\title{
Electromagnetic probes of a pure-glue initial state in nucleus-nucleus collisions at energies available at the CERN Large Hadron Collider
}

\author{
V. Vovchenko ${ }^{1,2,3}$, Iu. A. Karpenko ${ }^{4,5}$, M. I. Gorenstein ${ }^{1,4}$, \\ L. M. Satarov ${ }^{1,6}$, I. N. Mishustin ${ }^{1,6}$, B. Kämpfer ${ }^{7,8}$, and H. Stoecker ${ }^{1,2,9}$ \\ ${ }^{1}$ Frankfurt Institute for Advanced Studies, D-60438 Frankfurt, Germany \\ ${ }^{2}$ Johann Wolfgang Goethe Universität, D-60438 Frankfurt, Germany \\ ${ }^{3}$ Taras Shevchenko National University of Kiev, 03022 Kiev, Ukraine \\ ${ }^{4}$ Bogolyubov Institute for Theoretical Physics, 03680 Kiev, Ukraine \\ ${ }^{5}$ INFN - Sezione di Firenze, I-50019 Sesto Fiorentino (Firenze), Italy \\ ${ }^{6}$ National Research Center "Kurchatov Institute", 123182 Moscow, Russia \\ ${ }^{7}$ Helmholtz-Zentrum Dresden-Rossendorf, D-01314 Dresden, Germany \\ ${ }^{8}$ Technische Universität Dresden, Institut für Theoretische Physik, D-01062 Dresden, Germany \\ ${ }^{9}$ GSI Helmholtzzentrum für Schwerionenforschung GmbH, D-64291 Darmstadt, Germany
}




\begin{abstract}
Partonic matter produced in the early stage of ultrarelativistic nucleus-nucleus collisions is assumed to be composed mainly of gluons, and quarks and antiquarks are produced at later times. To study the implications of such a scenario, the dynamical evolution of a chemically nonequilibrated system is described by the ideal $(2+1)$-dimensional hydrodynamics with a time dependent (anti)quark fugacity. The equation of state interpolates linearly between the lattice data for the pure gluonic matter and the lattice data for the chemically equilibrated quark-gluon plasma. The spectra and elliptic flows of thermal dileptons and photons are calculated for central $\mathrm{Pb}+\mathrm{Pb}$ collisions at the CERN Large Hadron Collider energy of $\sqrt{s_{\mathrm{NN}}}=2.76 \mathrm{TeV}$. We test the sensitivity of the results to the choice of equilibration time, including also the case where the complete chemical equilibrium of partons is reached already at the initial stage. It is shown that a suppression of quarks at early times leads to a significant reduction of the yield of the thermal dileptons, but only to a rather modest suppression of the $p_{T}$-distribution of direct photons. It is demonstrated that an enhancement of photon and dilepton elliptic flows might serve as a promising signature of the pure-glue initial state.
\end{abstract}

PACS numbers: 12.38.Mh, 25.75.Cj, 47.75.+f 


\section{INTRODUCTION}

Strongly interacting matter with extremely high energy density can be created in the laboratory at the early stages of relativistic nucleus-nucleus $(\mathrm{A}+\mathrm{A})$ collisions. An important physical question is how the nonequilibrium initial system of two nucleon counter propagating flows of colliding nuclei transforms to a state of quarks and gluons in local thermodynamic equilibrium, i.e. to the quark-gluon plasma (QGP). The initial stage of $\mathrm{A}+\mathrm{A}$ collisions is presently described by different theoretical models ranging from simple parton cascades [1, 2], to more sophisticated string-parton models (UrQMD, PHSD, cf. [3] [5]), color glass condensate [6], coherent chromofields [7, 8], IP-Glasma [9] etc. It is usually assumed that strong nonequilibrium effects take place only during a very short proper time interval $\tau_{s} \sim 1 / Q_{s}$, where $Q_{s} \simeq 1 \div 2 \mathrm{GeV}$ is the so-called gluon saturation scale [10]. The idea that the gluonic components of colliding nucleons dominate in high energy collisions was originally put forward in Ref. [11]. It was motivated by the fact that the perturbative gluon-gluon cross sections are larger than the quark-antiquark ones. A two-step equilibration of QGP was proposed in [12 14] assuming that the gluon thermalization is accomplished already at the early proper time $\sim \tau_{s}$, while the quark-antiquark chemical equilibration proceeds until later times $\tau_{\text {th }}>\tau_{s}$. Reference [2] advocates that $\tau_{\text {th }}=5 \div 10 \mathrm{fm} / c$. Such a scenario for high energy A+A collisions was considered by several authors, see, e.g., Refs. [13, 15 24]. The pure glue initial scenario of $\mathrm{Pb}+\mathrm{Pb}$ collisions at CERN Large Hadron Collider (LHC) energies was recently discussed in Refs. [25, 26]. The particular aspect of entropy generation in the chemically nonequilibrated QGP has been addressed in [27].

In order to highlight possible signatures of the pure-glue initial scenario, below we describe the evolution of the QGP created in central A+A collisions using the $(2+1)$-dimensional boost-invariant hydrodynamics. In our approach the quark-antiquark fugacity is introduced to describe the QGP evolution in the absence of the chemical equilibrium. The main emphasis is put on electromagnetic probes (thermal photons and dileptons), which may carry an important information about the deconfined phase. This problem has been repeatedly addressed in the literature, see, e.g., [16, 17, 21 23], however, a definitive conclusion about the role of chemically nonequilibrium evolution is still missing. The new aspects of the present study include constructing the equation of state for chemically nonequilibrated QCD matter via an interpolation of the lattice data, as well as analyzing the impact of chemical nonequi- 
librium effects on the dilepton elliptic flow, and demonstrating the importance of the late 'hadronic' stage for the photon spectra.

The paper is organized as follows. In Sec. II we formulate the hydrodynamical model used in our calculations. The equation of state of a chemically nonequilibrated system is constructed by interpolating the lattice results between the pure gluon and the $(2+1)$-flavour QCD matter. In Sec. III we give some results concerning the space-time evolution of strongly interacting matter produced in central $\mathrm{A}+\mathrm{A}$ collisions at LHC energies. Spectra and elliptic flows of direct photons and thermal dileptons are analysed, respectively, in Sec. IV and V. Our conclusions are given in Sec. VI. Appendices A and B provide formulas for photon and dilepton rates, respectively.

\section{FORMULATION OF THE MODEL}

\section{A. Equations of motion}

We use a longitudinally boost-invariant $(2+1)$-dimensional ideal hydrodynamics to describe the evolution of the net baryon-free matter produced in the high-energy $\mathrm{A}+\mathrm{A}$ collisions. The equations of the relativistic hydrodynamics can be written as $(\hbar=c=1)$

$$
\frac{\partial T^{\mu \nu}}{\partial x^{\nu}}=0
$$

where

$$
T^{\mu \nu}=(\varepsilon+P) u^{\mu} u^{\nu}-P g^{\mu \nu}
$$

is the energy-momentum tensor, $u^{\mu}$ is the four-velocity, $\varepsilon$ and $P$ are the local rest-frame energy density and pressure, respectively, and $g^{\mu \nu}$ is the metric tensor with $g^{00}=1$ in Cartesian coordinates $(t, x, y, z)$, with $z$ oriented along the beam axis. Below we use the curvilinear light-cone coordinates $(\tau, x, y, \eta)$, where $\tau=\sqrt{t^{2}-z^{2}}$ is the proper time and $\eta=\frac{1}{2} \ln \frac{t+z}{t-z}$ is the space-time rapidity. In the case of the longitudinal boost-invariant $(2+1)$-dimensional flow one can represent the the fluid's four-velocity as [16, 29]

$$
u^{\mu}=\gamma_{\perp}\left(\cosh \eta, \boldsymbol{v}_{\perp}, \sinh \eta\right),
$$

where $\boldsymbol{v}_{\perp}$ is the transverse velocity in the symmetry plane $z=0$ and $\gamma_{\perp}=\left(1-v_{\perp}^{2}\right)^{-1 / 2}$ stands for the transverse Lorentz factor. To solve Eq. (11) one needs the equation of state 
(EoS), i.e., a relation connecting $P$ and $\varepsilon$. For chemically nonequilibrated matter considered in this paper, $P=P(\varepsilon, \lambda)$, where $\lambda$ is the (anti)quark fugacity. In principle, one should also solve additional rate equations, defining the evolution of $\lambda$, as done, e.g., in [15, 16, 23, 28]. Instead, a simple analytic parametrization for $\lambda$ as a function of the proper time is used in our study (see the next section).

It is useful to introduce the local proper time $\tau_{P}$ of a fluid cell element. Its space-time dependence is determined by the equations

$$
u^{\mu} \partial_{\mu} \tau_{P}=1, \quad \tau_{P}\left(\tau_{0}, x, y, \eta\right)=\tau_{0}
$$

where the parameter $\tau_{0}$ corresponds to initial longitudinal proper time of the hydrodynamic expansion. Equation (41) must be solved simultaneously with Eq. (11). In general, $\tau_{P}$ is smaller than the 'global' time $\tau$ due to the presence of non-zero transverse flow. In the limiting case of the one-dimensional longitudinal Bjorken expansion [30], one has $v_{\perp}=0$ and, consequently, $\tau_{P}=\tau$.

\section{B. Equation of state of chemically nonequilibrium QCD matter}

We use the lattice QCD calculations for the EoS of the strongly interacting matter in two limiting cases: (1) the chemically equilibrated QCD matter [32, 33] and (2) the SU(3) gluodynamics without (anti)quarks [34, 35]. In the following we denote these cases as FQ (Full QCD) and PG (Pure Glue), respectively. The FQ case corresponds to the $(2+1)$-flavour QCD calculations which predict the crossover-type transition at $T \sim 155 \mathrm{MeV}$. The PG calculation provides a first-order phase transition at $T=T_{c} \simeq 270 \mathrm{MeV}$. The temperature dependencies of the pressure and energy density for FQ and PG scenarios are exhibited in Fig. 1. Larger values of $P$ and $\varepsilon$ in the FQ calculation appear due to the contribution of quark-antiquark degrees of freedom. Note the discontinuity of $\varepsilon(T)$ at $T=T_{c}$ in the PG case. Very small values of $P$ and $\varepsilon$ at $T<T_{c}$ in the $\mathrm{PG}$ matter originate from large masses of glueballs $\left(M_{g} \gg T_{c}\right)$ which are the constituents of the confined phase [35].

The suppression of the quark and antiquark densities as compared to their equilibrium values at given temperature is characterized by the (anti)quark fugacity $\lambda$ (for details, see Ref. [27]). Generalizing the lattice EoS for the chemically nonequilibrium case with $\lambda<1$ is not a straightforward task. We obtain the $P$ and $\varepsilon$ values at fixed $T$ and $\lambda$ by a linear 

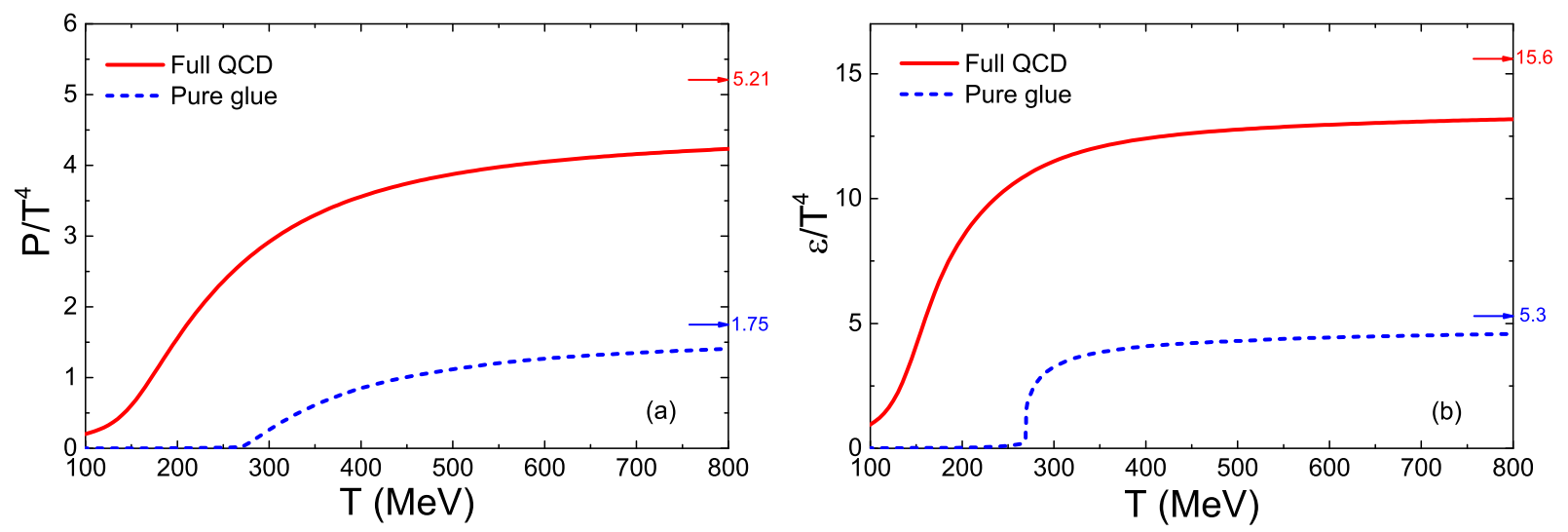

FIG. 1: (Color online) Temperature dependence of the scaled pressure (a) and the scaled energy density (b) obtained in lattice QCD calculations of Refs. [32, 35]. The solid and dashed lines correspond to the FQ $\left(N_{f}=2+1\right)$ and $\mathrm{PG}\left(N_{f}=0\right)$ cases, respectively. The horizontal arrows indicate the asymptotic (Stefan-Boltzmann) values of $P / T^{4}$ and $\varepsilon / T^{4}$ at large temperatures.

interpolation (LI) between the PG and FQ cases ${ }^{1}$ :

$$
\begin{aligned}
P(T, \lambda) & =\lambda P_{\mathrm{FQ}}(T)+(1-\lambda) P_{\mathrm{PG}}(T), \\
\varepsilon(T, \lambda) & =\lambda \varepsilon_{\mathrm{FQ}}(T)+(1-\lambda) \varepsilon_{\mathrm{PG}}(T) .
\end{aligned}
$$

After excluding the temperature variable in Eqs. (5) and (6), one gets the relation $P=$ $P(\varepsilon, \lambda)$ which is used in hydrodynamic simulations. The limits $\lambda=0$ and $\lambda=1$ correspond to the thermodynamic functions of the PG and FQ matter, respectively. Note that the linear $\lambda$-dependence of $P$ and $\varepsilon$ is a characteristic feature of the ideal gas of massless gluons and (anti)quarks studied in Refs. [26, 27].

It is interesting that the $\varepsilon$-dependence of the pressure needed for the hydrodynamical calculations appears to be rather similar in PG and FQ matter. This is shown in Fig. 2. The pressure values corresponding to EoS-LI will change from $P=P_{\mathrm{PG}}(\varepsilon)$ at the initial stage of the $\mathrm{A}+\mathrm{A}$ collision to $P=P_{\mathrm{FQ}}(\varepsilon)$ during the later stage of chemical equilibration ${ }^{2}$. As follows from Fig. 2, both equations of state show an almost linear $P(\varepsilon)$ dependence in the considered energy density range, but they are both softer than the EoS of the ideal gas of massless partons.

\footnotetext{
1 For brevity, we denote this equation of state as EoS-LI.

2 Possible supercooling phenomena may change this behavior.
} 


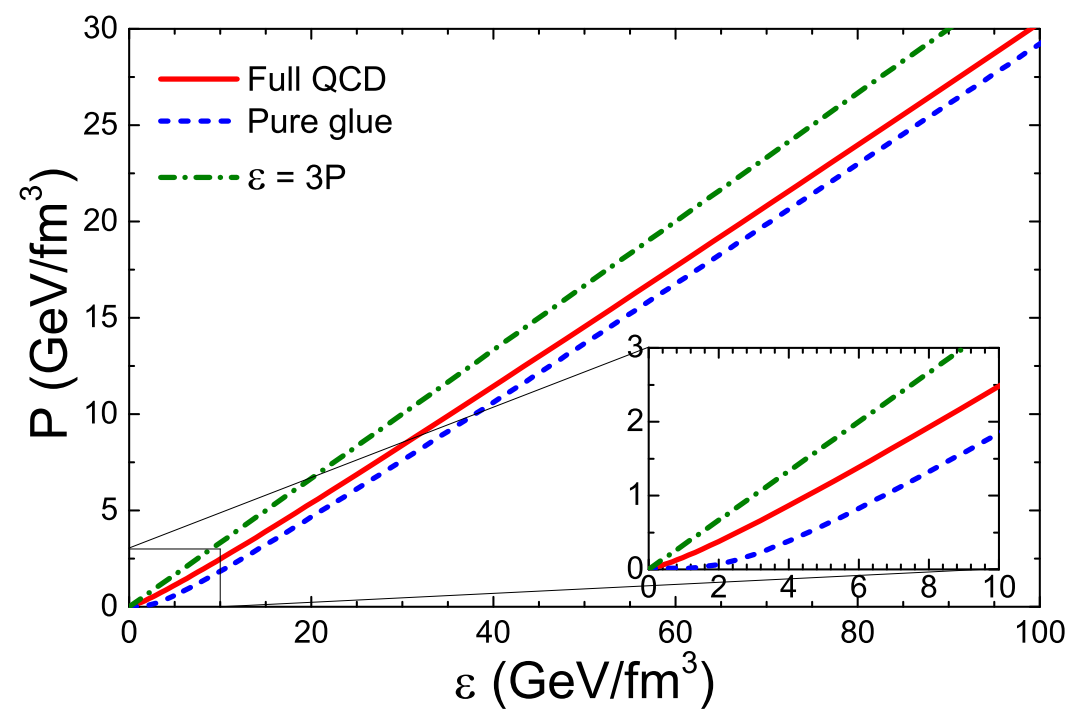

FIG. 2: (Color online) Pressure as a function of energy density in FQ (solid curve) and PG (dashed curve) cases obtained in lattice calculations [32, 35]. Additionally, the $P=\varepsilon / 3$ dependence for the ultrarelativistic ideal gas is shown by the dash-dotted line. The inset zooms into the region of smaller energy densities.

Using Eqs. (5) and (6) and basic thermodynamic identities, one can calculate the total density of (anti)quarks $n_{q}$ and the entropy density $s$. The following relations are obtained

$$
\begin{aligned}
n_{q}(T, \lambda) & =\frac{\lambda}{T}\left(P_{\mathrm{FQ}}-P_{\mathrm{PG}}\right), \\
s(T, \lambda) & =\lambda s_{\mathrm{FQ}}(T)+(1-\lambda) s_{\mathrm{PG}}(T)-n_{q}(T, \lambda) \ln \lambda .
\end{aligned}
$$

The two-dimensional plots of $P$ and $\varepsilon$ for the chemically nonequilibrated QCD are shown in Fig. 3. The EoS-LI contains the first-order phase transition at $T_{c}=270 \mathrm{MeV}$. The latent heat of this transition depends on $\lambda$, and it goes to zero at $\lambda \rightarrow 1$.

Below we assume that at $\tau=\tau_{0}$ the initial (anti)quark densities vanish in all cells and gluons are in thermal and chemical equilibrium. Similarly to Refs. [26, 27] we postulate that $\lambda$ is an explicit function of the local proper time $\tau_{P}$ which increases from $\lambda=0$ at $\tau_{P}=\tau_{0}$ to $\lambda=1$ at $\tau_{P}-\tau_{0} \rightarrow \infty$. The following simple parametrization is used:

$$
\lambda\left(\tau_{P}\right)=1-\exp \left(\frac{\tau_{0}-\tau_{P}}{\tau_{*}}\right),
$$

where $\tau_{*}$ is a model parameter characterizing the quark chemical equilibration time. There are different estimates for $\tau_{*}$ in the literature ranging from $\tau_{*} \sim 1 \mathrm{fm} / c$ [31] to 

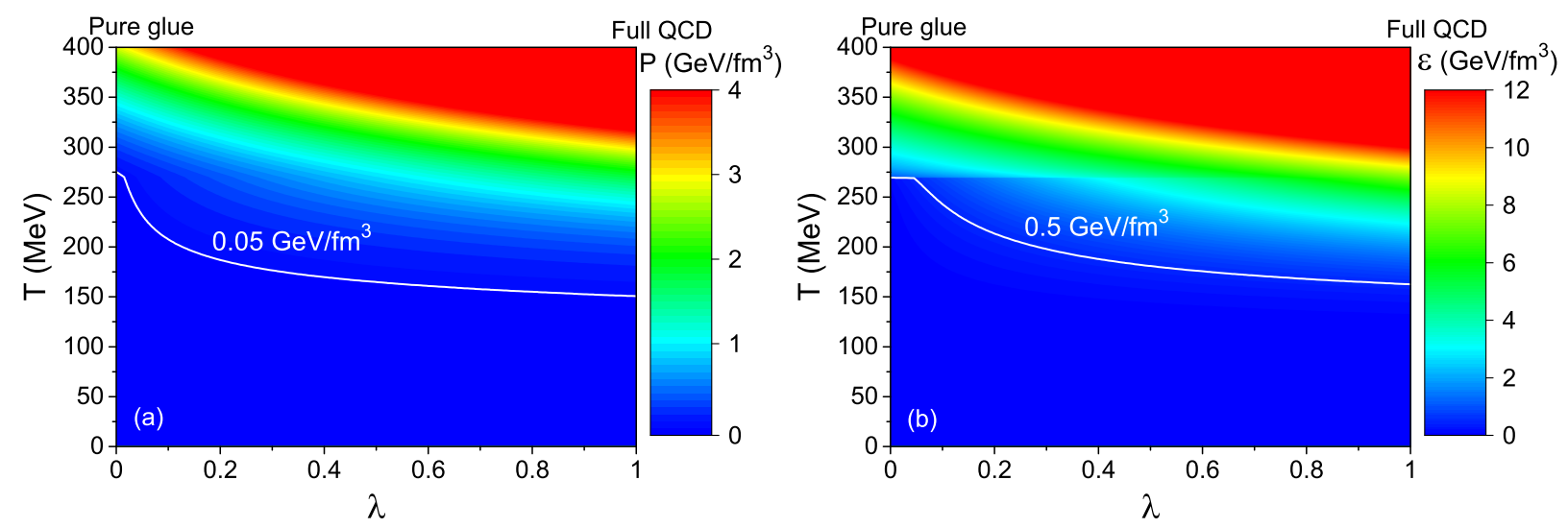

FIG. 3: (Color online) Contour plots of pressure (a) and energy density (b) for chemically non-equilibrated QCD calculated from Eqs. (5) and (6). The white lines show contours $P=0.05 \mathrm{GeV} / \mathrm{fm}^{3}$ (a) and $\varepsilon=0.5 \mathrm{GeV} / \mathrm{fm}^{3}(\mathrm{~b})$.

$\tau_{*} \sim 5 \mathrm{fm} / c[2]$. Note that $\tau_{*}=0$ corresponds to the instantaneous chemical equilibration of quarks and gluons.

In our calculations we assume that gluons are always in thermal and chemical equilibrium immediately from the beginning of the hydro expansion. This assumption can be relaxed by modeling the chemical non-equilibrium of gluons by using the time dependent gluon fugacity $\lambda_{g}$, with a different (smaller) relaxation time $\tau_{g}$ for gluons compared to quarks. The calculations can be made even more realistic by introducing additional rate equations describing the space-time evolution of quark and gluon densities. However, this would require some new assumptions. In particular, the introduction of the chemical non-equilibrium for gluons would require modifications to the equation of state. Calculations employing the rate equations [19, 21, 31], as well as those employing the microscopic parton cascade [25], indicate that the time evolution of the gluon fugacity is not completely trivial, and may even be non-monotonic. For our analysis it is most important that, at the early stages, the gluon fugacity is still significantly larger than the (anti)quark fugacity. Thus, in the present work we only consider the undersaturation of quarks, but not of gluons.

\section{Initial conditions}

We consider $\mathrm{Pb}+\mathrm{Pb}$ collisions at the LHC with center-of-mass (c.m.) energy per nucleon pair $\sqrt{s_{N N}}=2.76 \mathrm{TeV}$. In our calculations we choose $\tau_{0}=0.1 \mathrm{fm} / \mathrm{c}$ as the initial time 
of the hydrodynamic evolution. It is assumed that there is no initial transverse flow, i.e., $\boldsymbol{v}_{\perp}\left(\tau_{0}, x, y\right)=0$, and the initial energy density profile is proportional to the linear combination of the transverse distributions of wounded nucleons and of binary collisions taken from the event-averaged Monte Carlo Glauber model as implemented in the GLISSANDO code [36]. The coefficient of proportionality in the initial $\varepsilon$-profile is fixed to reproduce the observed hadron spectra within the simulation assuming chemical equilibrium with the full QCD EoS for a given centrality interval (see Ref. [37] for details). We use the same initial energy density profile in the present calculations for the chemical nonequilibrium case.

It is also assumed that initially the fugacity $\lambda$ of (anti)quarks is zero, i.e. the initial state is purely gluonic. In our model this is realized by setting the initial local proper time of each fluid element equal to $\tau_{0}$, i.e. $\tau_{P}\left(\tau_{0}, x, y, \eta\right)=\tau_{0}$.

\section{NUMERICAL RESULTS OF HYDRODYNAMIC SIMULATIONS}

Equations (11) and (4) are solved using the (2+1)-dimensional version of the vHLLE hydro code [38]. The EoS tables $P=P(\varepsilon, \lambda)$ for hydrodynamic simulations were prepared as described in Sec. IIB. We consider the 0-20\% and 20-40\% central Pb+Pb collisions.

The density plot of the quark fugacity $\lambda$ in the $x-\tau$ plane is given in Fig. 4 a. The dashed line shows the isotherm $T=155 \mathrm{MeV}$ which presumably corresponds to the hadronization hypersurface. One can see that typical lifetimes of the deconfined phase in the considered reaction do not exceed $10 \mathrm{fm} / c$. In Fig. 4a one observes that deviations from chemical equilibrium $(\lambda \lesssim 0.9)$ survive up to the hadronization stage. As discussed in Ref. [27] this may lead to a suppression of (anti)baryon-to-pion ratios observed [39] for the considered reaction. Note that $\lambda$ evolves with $\tau$ in Fig. 4 a even in the large $x$ regions where there is virtually no matter, which results from applying the Eq. (9). In reality, of course, the values of $\lambda$ for very dilute and cold fluid elements are irrelevant and should be ignored.

Figure 4]b shows the density plot of the temperature in the coordinates $(x, \tau)$. The solid and dashed curves correspond to $\tau_{*}=5 \mathrm{fm} / c$ and $\tau_{*}=0$, respectively. One can see that the chemically undersaturated matter is hotter as compared to the equilibrium case $(\lambda=1)^{3}$. This is a consequence of reduced number of degrees of freedom in such a medium.

\footnotetext{
3 Note that in both cases we take the same profile of the energy density at $\tau=\tau_{0}$.
} 

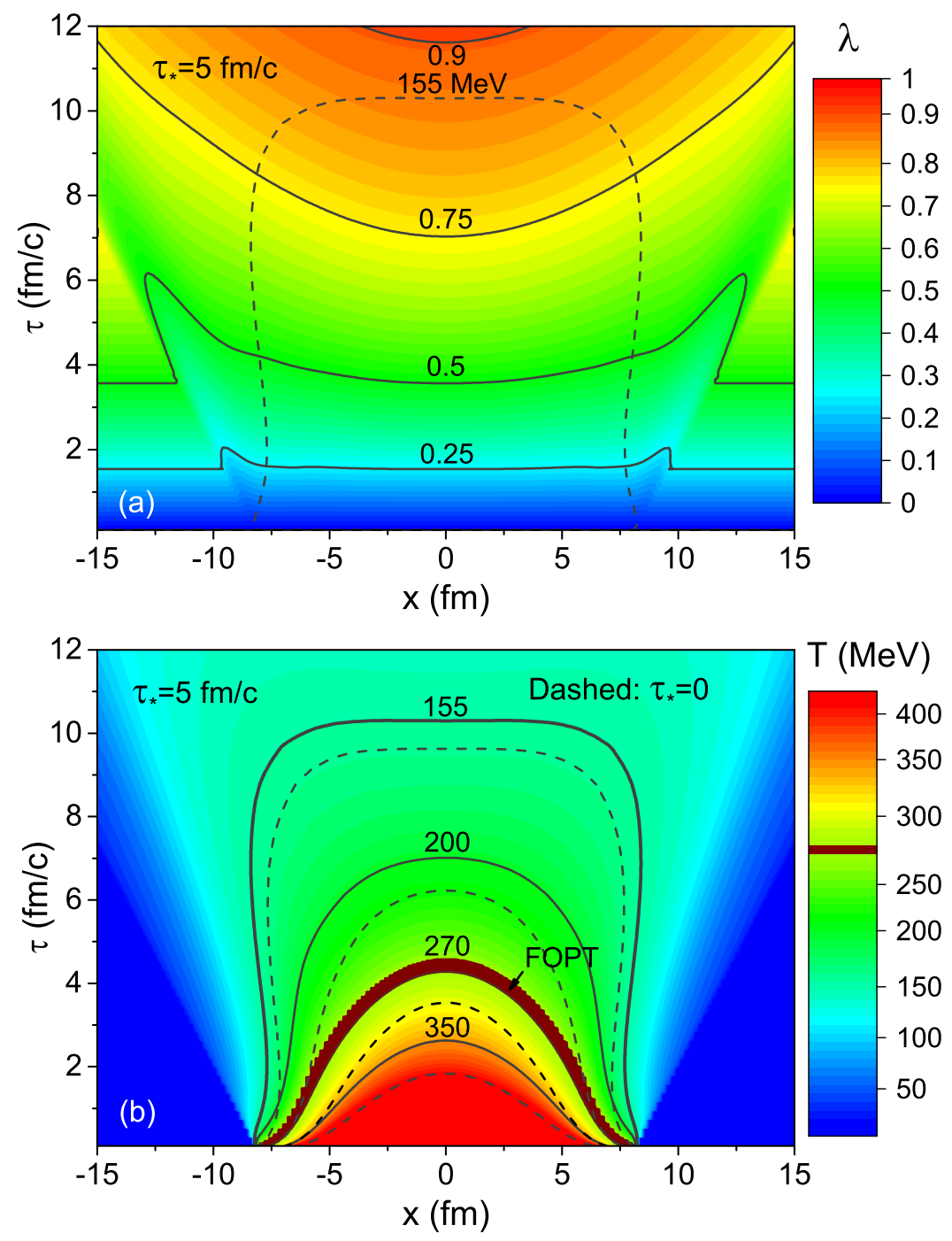

FIG. 4: (Color online) Contour plots of the quark fugacity (a) and temperature (b) in the $x-\tau$ plane for the $0-20 \%$ most central $\mathrm{Pb}+\mathrm{Pb}$ collisions at $\sqrt{s_{N N}}=2.76 \mathrm{TeV}$. The solid curves show contours of $\lambda$ and $T$ (in units of $\mathrm{MeV}$ ). The dashed line in (a) corresponds to the isotherm $T=$ $155 \mathrm{MeV}$. The dark region labeled by FOPT corresponds to the mixed-phase region of the firstorder phase transition at $T=T_{c} \simeq 270 \mathrm{MeV}$. The dashed curves in (b) depict isotherms calculated for equilibrium matter with $\lambda=1$.

According to Fig. 4 $\mathrm{b}$, typical lifetimes of the mixed phase are rather short, they do not exceed $0.5 \mathrm{fm} / c$. This is at variance with calculations in the $(1+1)$ dimensional hydrodynamics which predict [40] much larger lifetimes of the mixed phase within the chemically equilibrated bag model. Therefore, the account of transverse expansion is rather important.

Figure 5 shows the evolution of the temperature in the central cell $(x, y, z)=0$ for $\tau_{*}=0$ 


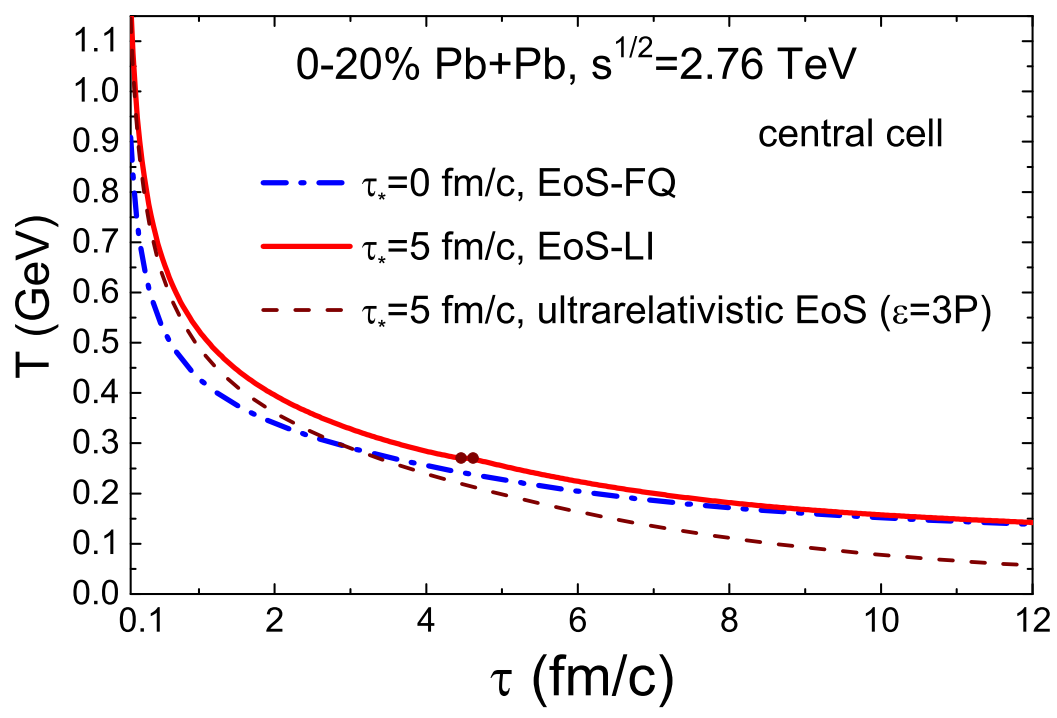

FIG. 5: (Color online) Temperature in the central cell as a function of proper time for the $0-20 \%$ central $\mathrm{Pb}+\mathrm{Pb}$ collisions at $\sqrt{s_{N N}}=2.76 \mathrm{TeV}$. The dashed and solid curves are calculated for $\tau_{*}=0$ and $\tau_{*}=5 \mathrm{fm} / c$, respectively. The short section between filled dots on the solid curve corresponds to mixed-phase states of the confinement phase transition. The dash-dotted curve is for $\tau_{*}=5 \mathrm{fm} / c$ assuming the ideal gas EoS.

and $\tau_{*}=5 \mathrm{fm} / c$. In the second case, $\tau_{*}=5 \mathrm{fm} / c$ we compare the calculations for EoSLI (solid line) and for the ideal gas of massless partons [27] (dashed line). One can see significant differences between these two calculations at late times.

It is evident that the entropy will grow in the course of chemical equilibration. This was demonstrated in Ref. [27] within the purely longitudinal Bjorken hydrodynamics. Here we present a similar analysis within the (2+1)-dimensional hydrodynamical model. The total amount of entropy flowing through a space-time hypersurface $\sigma^{\mu}$ can be evaluated as [40]:

$$
S=\int d \sigma^{\mu} u_{\mu} s .
$$

Here $s$ is the entropy density and $d \sigma^{\mu}$ is the element of a space-time hypersurface which we choose below ${ }^{4}$ as the surface of constant proper time $\tau$. Using Eq. (3), one can show that $d \sigma^{\mu} u_{\mu}=\gamma_{\perp} \tau d^{2} x_{\perp} d \eta$ for such a hypersurface. Substituting this relation into Eq. (10) leads to the following expression for the total entropy per unit space-time rapidity in

\footnotetext{
4 In the case of chemical equilibrium $S$ is constant and does not depend on the choice of a hypersurface.
} 
the $(2+1)$-dimensional hydrodynamics

$$
\frac{d S(\tau)}{d \eta}=\tau \int d^{2} x_{\perp} \gamma_{\perp}\left(\tau, \boldsymbol{x}_{\perp}\right) s\left(\tau, \boldsymbol{x}_{\perp}\right)
$$

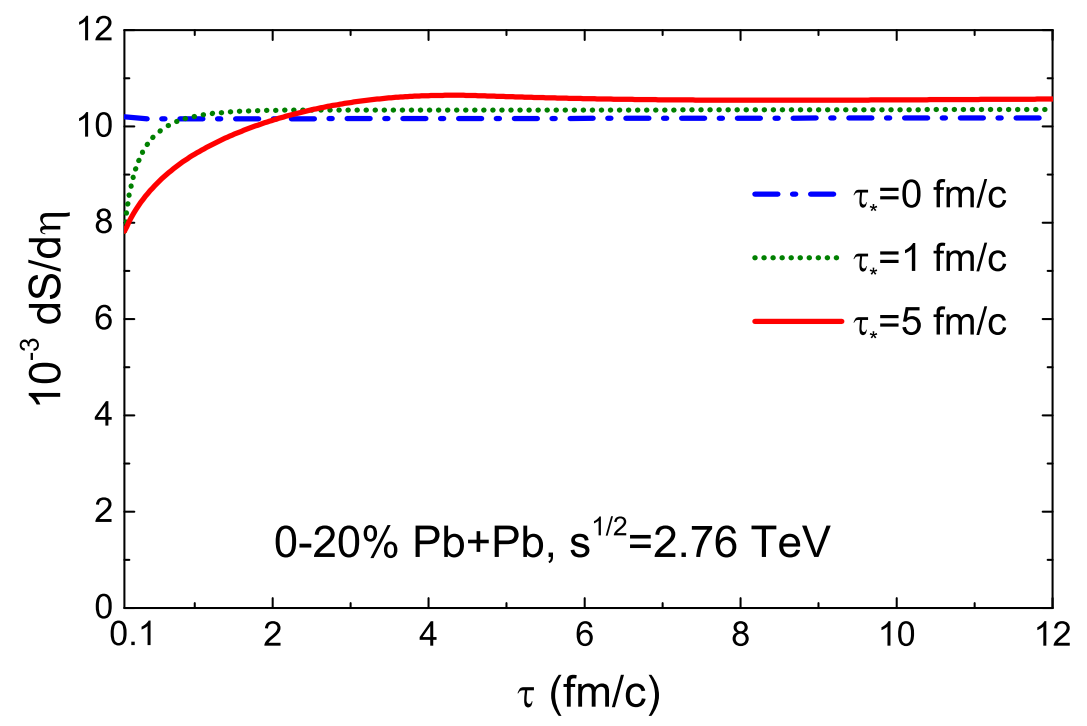

FIG. 6: (Color online) Total entropy per unit space-time rapidity as a function of proper time $\tau$ for the $0-20 \%$ central $\mathrm{Pb}+\mathrm{Pb}$ collisions at $\sqrt{s_{N N}}=2.76 \mathrm{TeV}$. The dash-dotted, dotted and solid curves correspond to the parameters $\tau_{*}=0,1$ and $5 \mathrm{fm} / c$, respectively.

The results of the entropy calculations for the $0-20 \%$ central $\mathrm{Pb}+\mathrm{Pb}$ collisions are shown in Fig. 6. At $\tau_{*}=5 \mathrm{fm} / \mathrm{c}$ the relative increase of the entropy is about $30 \%$. Approximately the same relative increase has been obtained in [27] within a one-dimensional Bjorken-like calculation for the ideal gas EoS. Note, that more consistent calculations for nonzero $\tau_{*}$ would require renormalizing the initial energy density profiles to obtain the same final pion multiplicities as in the equilibrium case. The asymptotic values of $d S / d \eta$ for different choices of $\tau_{*}$ in Fig. [6 will be then the same.

\section{DIRECT PHOTON EMISSION}

The emission of direct ${ }^{5}$ photons from expanding matter created in relativistic $\mathrm{A}+\mathrm{A}$ collisions has several components [41, 42]: a) 'prompt' photons from binary collisions of initial

5 By direct photons we denote the 'non-cocktail' photons i.e. those which are not produced in decays of $\pi^{0}, \eta, \rho, \eta^{\prime}$, and $\phi$ mesons in the final stage of the reaction. 
nucleons, b) 'thermal' photons from the high-temperature deconfined phase, c) direct photons from the low-temperature hadronic phase. The contribution of prompt photons becomes dominant at large transverse momenta. As we will see below, this greatly reduces the sensitivity of photon $p_{T}$-spectra to chemical nonequilibrium effects. However, the situation with transverse flows of photons is different because of low azimuthal anisotropy of prompt photons. Note that the ALICE experiments [43] reveal large elliptic flows of direct photons, which still can not be explained in the chemically equilibrium scenario [41].

Within the leading order approximation in the strong coupling constant, the following sources of thermal photon production in the deconfined matter are dominant [44]:

1) QCD Compton scattering $(A+g \rightarrow A+\gamma$, where $A=q, \bar{q})$,

2) quark-antiquark annihilation $(q+\bar{q} \rightarrow g+\gamma)$,

3 ) bremsstrahlung reactions $(A+B \rightarrow A+B+\gamma$, where $A=q, \bar{q}$ and $B=q, \bar{q}, g)$,

4) 'off-shell' $q \bar{q}$-annihilation with rescatterings of (anti)quark on another parton in the initial state $^{6}$. It is clear that photons can not be produced in a pure glue matter without charged (anti)quark partons.

Let us consider the invariant photon production rate (PPR) in the chemically undersaturated quark-gluon plasma (uQGP) with the temperature $T$ and the quark fugacity $\lambda$. Below we denote this quantity as $\Gamma(\widetilde{E}, T, \lambda)$, where $\widetilde{E}$ is the photon energy in the rest frame of the fluid element. The limiting case of complete chemical equilibrium $(\lambda=1)$ is considered in Appendix A. We use the analytic parametrization for $\Gamma(\widetilde{E}, T)=\Gamma(\widetilde{E}, T, 1)$ suggested in Ref. [44]. Equations (A1)-(A2) give the explicit expressions for $\Gamma_{i}(\widetilde{E}, T)$, which are the PPR of processes $i=1,2$ in the chemically equilibrated QGP.

To calculate PPR for arbitrary $\lambda$ we introduce the additional suppression factor $\lambda$ for each quark and antiquark [26, 27] in initial states of the processes 1-4. In particular, the rates of the processes 1 and 2 will be suppressed by the factors $\lambda$ and $\lambda^{2}$, respectively. An analogous procedure for the processes 3 and 4 is not trivial, as the contribution of partons $B=g$ is not suppressed as compared to $B=q, \bar{q}$. Similar to Ref. [22], we apply two different

6 According to Ref. [45], the next-to-leading order corrections to the rate of photon production in equilibrium QGP do not exceed $20 \%$. 

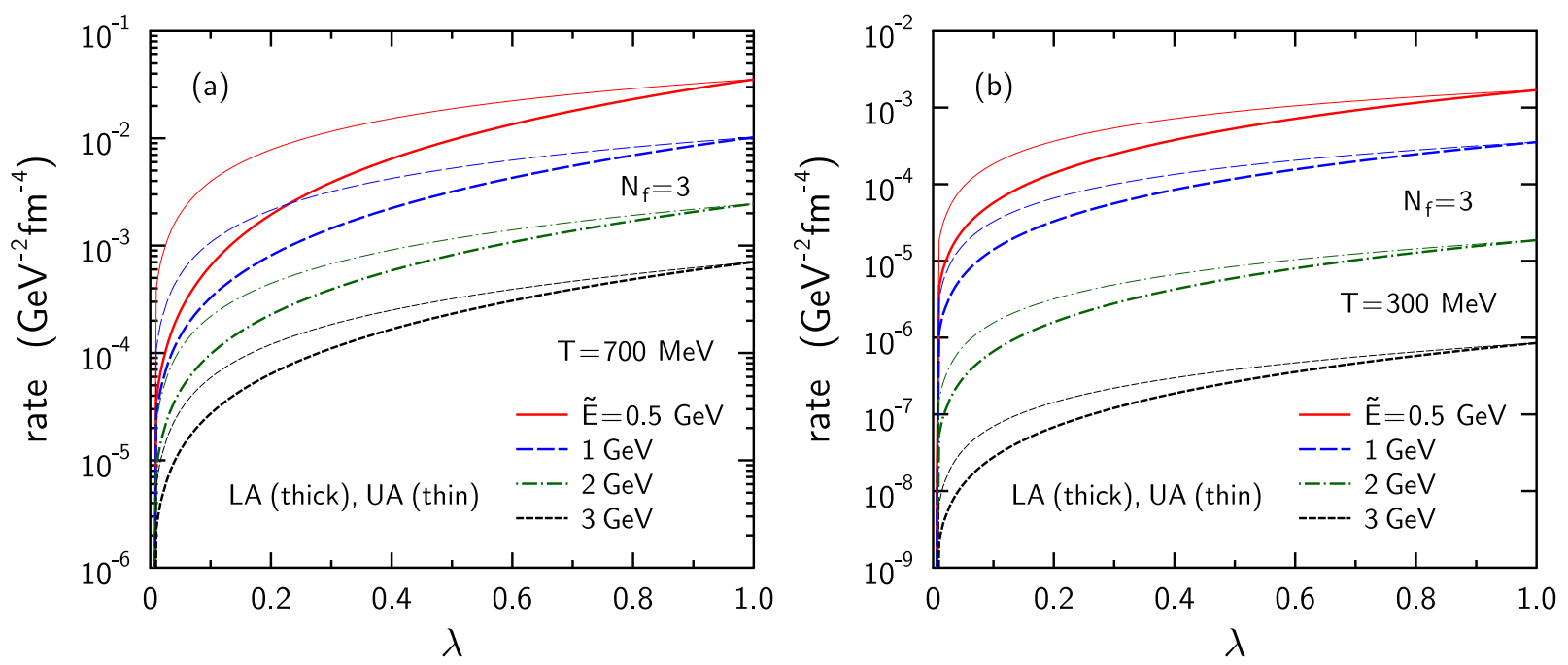

FIG. 7: (Color online) Thermal photon production rates in uQGP as functions of quark fugacity $\lambda$ at temperatures $T=700$ (a) and 300 (b) $\mathrm{MeV}$ for different values of rest-frame photon energy $\widetilde{E}$. Thick and thin lines are calculated by using Eq. (12) and (13), respectively.

approximations for $\Gamma(\widetilde{E}, T, \lambda)$ :

$$
\begin{array}{ll}
\text { LA: } & \Gamma(\widetilde{E}, T, \lambda)=\lambda \Gamma_{1}(\widetilde{E}, T)+\lambda^{2}\left[\Gamma(\widetilde{E}, T)-\Gamma_{1}(\widetilde{E}, T)\right], \\
\mathrm{UA}: & \Gamma(\widetilde{E}, T, \lambda)=\lambda^{2} \Gamma_{2}(\widetilde{E}, T)+\lambda\left[\Gamma(\widetilde{E}, T)-\Gamma_{2}(\widetilde{E}, T)\right],
\end{array}
$$

where $\Gamma(\widetilde{E}, T)$ is calculated by using Eqs. (A4 - (A7). It is clear that the approximation LA (UA) underestimates (overestimates) the "exact" photon production rate in uQGP. The results of PPR calculations using Eqs. (12) and (13) are shown in Fig. 7 for several values of $\widetilde{E}$ and $T$. One can see that the difference between the parametrizations LA and UA increases with $T$. Note that large temperatures correspond to early stages of a heavy-ion collision, when the values of $\lambda$ are rather small in the pure glue initial scenario [26].

In our case of a boost invariant $(2+1)$-dimensional expansion the invariant yield of thermal photons is calculated as

$$
\frac{d N_{\gamma}^{\text {th }}}{d^{2} p_{T} d Y}=\int d^{2} x_{T} \int_{\tau_{0}}^{+\infty} d \tau \tau \int_{-\infty}^{+\infty} d \eta \Gamma(\widetilde{E}, T, \lambda) \theta\left(T-T_{f}\right)
$$

where $p_{T}$ is the transverse momentum of the photon, $Y$ is its longitudinal rapidity, $\widetilde{E}=$ $\gamma_{\perp} p_{T}\left[\cosh (Y-\eta)-v_{x} \cos \varphi-v_{y} \sin \varphi\right]$ ( $\varphi$ is the angle between $\boldsymbol{p}_{T}$ and the reaction plane), $\theta(x)=[1+\operatorname{sgn}(x)] / 2$, and $T_{f}$ is the minimum temperature, i.e. radiation from fluid cells 
with $T>T_{f}$ is considered only.
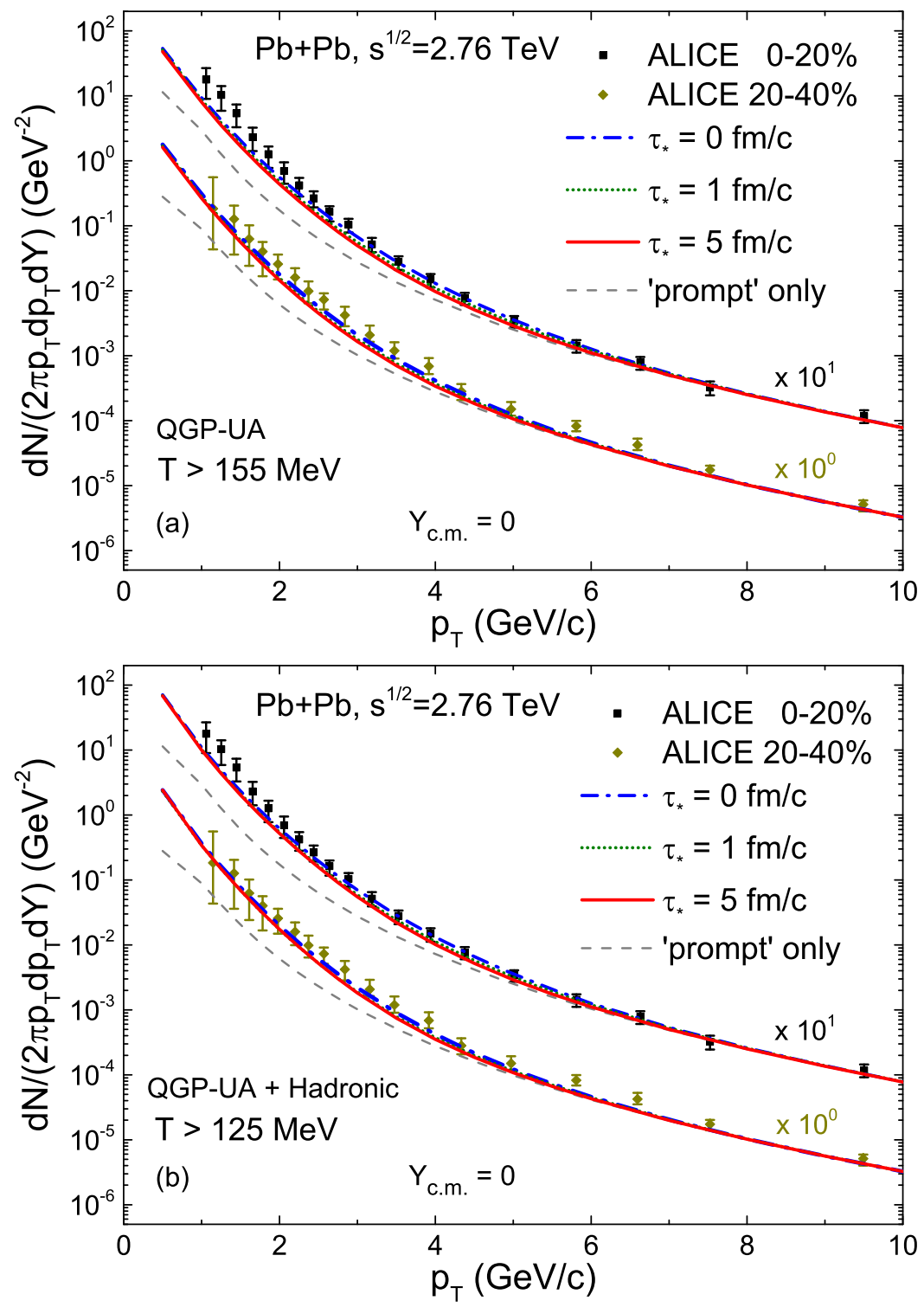

FIG. 8: (Color online) Spectra of direct photons in the 0-20\% central $\mathrm{Pb}+\mathrm{Pb}$ collisions at $\sqrt{s_{N N}}=$ $2.76 \mathrm{TeV}$ calculated by using Eq. (14) with the cutoff temperatures $T_{f}=155$ (a) and 125 (b) MeV. At $T>155 \mathrm{MeV}$ the uQGP photon production rate given by Eq. (13) is used while for lower temperatures the hadronic PPR is employed. The dash-dotted, dotted and solid curves correspond to $\tau_{*}=0,1$ and $5 \mathrm{fm} / c$, respectively. Dots with error bars show the experimental data [46].

As mentioned above, spectra of direct photons include, in addition to the thermal component, also the contribution of prompt photons from initial collisions of nucleons in cold initial nuclei. This contribution is usually obtained by using the perturbative QCD cal- 
culations of photon production in a single $p p$-collision at the same $\sqrt{s_{N N}}$. The obtained photon yield is scaled by the average number of nucleon collisions for a given centrality class. Below we use the prompt photon spectra in central $\mathrm{Pb}+\mathrm{Pb}$ collisions at $\mathrm{LHC}$ reported in Refs. [43, 46]. According to our calculations, the contribution of prompt photons in such reactions becomes dominant at high transverse momenta $p_{T} \gtrsim 5 \mathrm{GeV} / c$. Unfortunately, this greatly reduces the sensitivity of combined thermal and prompt photon $p_{T}$-spectra to the EoS and to parameters of chemical nonequilibrium.

Figure 8 a shows our results for the direct photon spectrum in the $0-20 \%$ central $\mathrm{Pb}+\mathrm{Pb}$ collisions at $\sqrt{s_{N N}}=2.76 \mathrm{TeV}$, calculated using the PPR from Eqs. (12) and (13) with the cut-off temperature of $T_{f}=155 \mathrm{MeV}$. We have checked that the LA parametrization of thermal photon emission, Eq. (12), gives only several percent lower yields as compared to the alternative UA choice. Therefore, we present here only the results based on Eq. (13). To estimate contributions of thermal photon emission from the late (hadronic) stages of the reaction, we additionally perform calculations for the lower cut-off temperature of $125 \mathrm{MeV}$, shown in Fig. 8fb. In this case, for temperatures $T<155 \mathrm{MeV}$ we use the parametrized PPR in the hadronic phase, which includes contributions of the the in-medium $\rho$ mesons [47], strange mesons [48], and the $\pi \pi$-bremsstrahlung [47]. The consistency of our approach is provided by the fact that hadronic and QGP rates are very similar in the vicinity of the crossover temperature, as demonstrated in [41,49]. In our calculations we use the chemically equilibrium rates of photon emission from the confined phase. In principle, the hadronic densities may be reduced due to the suppression of constituent (anti)quarks with fugacities $\lambda<1$ (see the related discussion in Ref. [27]). However, as one can see in Fig. 4, at these low temperatures the $\lambda$-values are already close to unity. Having in mind that introducing the suppressed hadronic rates will require additional assumptions and that its effect is expected to be small, we do not implement the corresponding modification of hadronic PPR in the present paper. Comparison of Figs. 8 $\mathrm{a}$ and b shows that thermal photon emission from the low-temperature stage $T \lesssim 155 \mathrm{MeV}$ gives only a slight change of the yield at intermediate $p_{T}=2 \div 6 \mathrm{GeV} / \mathrm{c}$. On the other hand, such emission more noticeably increases the photon yield at $p_{T} \lesssim 2 \mathrm{GeV} / c$. Including this additional hadronic source of thermal photons leads to a somewhat better agreement with the observed data. We note that PPR from hadronic phase are presently not constrained very well. Thus, additional studies in that direction are needed. 
The direct photon production in $\mathrm{Pb}+\mathrm{Pb}$ collisions at $\mathrm{LHC}$ has been considered in various theoretical models which include relativistic ideal [50, 51] or viscous [41] hydrodynamics, and the PHSD off-shell transport approach [52]. These studies describe experimental $p_{T^{-}}$-spectra of photons with a similar quality. Thus, as noted in Ref. [46], the present uncertainties in the ALICE photon data do not allow to discriminate between various models and scenarios.
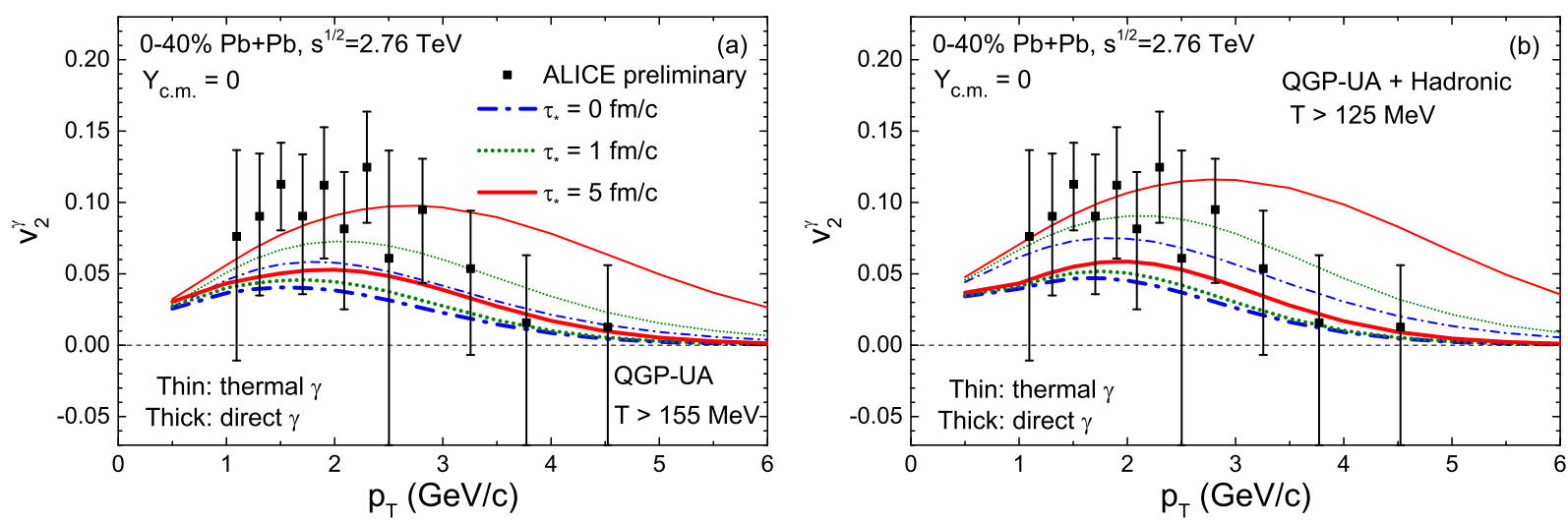

FIG. 9: (Color online) Elliptic flow $v_{2}^{\gamma}$ of direct photons as a function of transverse momentum $p_{T}$ in the $0-40 \%$ central $\mathrm{Pb}+\mathrm{Pb}$ collisions at $\sqrt{s_{N N}}=2.76 \mathrm{TeV}$ calculated with the cutoff temperatures $T_{f}=155$ (a) and 125 (b) MeV. The dash-dotted, dotted and solid lines correspond to $\tau_{*}=0,1$ and $5 \mathrm{fm} / c$, respectively. Thick (thin) curves are calculated with (without) the contribution of prompt photons in Eq. (15). Experimental data are taken from Ref. [43].

The photon elliptic flow $v_{2}^{\gamma}\left(p_{T}\right)$ is calculated by

$$
v_{2}^{\gamma}\left(p_{T}\right)=\frac{\int_{0}^{2 \pi} d \varphi \frac{d N_{\gamma}}{d^{2} p_{T} d Y} \cos (2 \varphi)}{\int_{0}^{2 \pi} d \varphi \frac{d N_{\gamma}}{d^{2} p_{T} d Y}} .
$$

The photon spectrum, entering this equation includes both thermal and prompt components. We assume that prompt photons are azimuthally symmetric. Therefore, they contribute only to the denominator of Eq. (15) reducing $v_{2}^{\gamma}$ at large $p_{T}$.

The results for direct photon elliptic flow in the $0-40 \%$ central $\mathrm{Pb}+\mathrm{Pb}$ collisions at $\sqrt{s_{N N}}=2.76 \mathrm{TeV}$ are shown in Figs. 9]a and b. One can see that the initial undersaturation of quarks leads to a noticeable enhancement of $v_{2}^{\gamma}$. The comparison of thick and thin lines shows that this enhancement is significantly reduced due to the presence of prompt photons ${ }^{7}$.

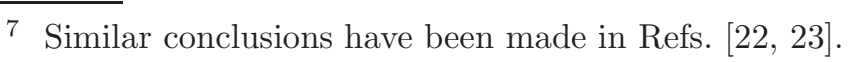




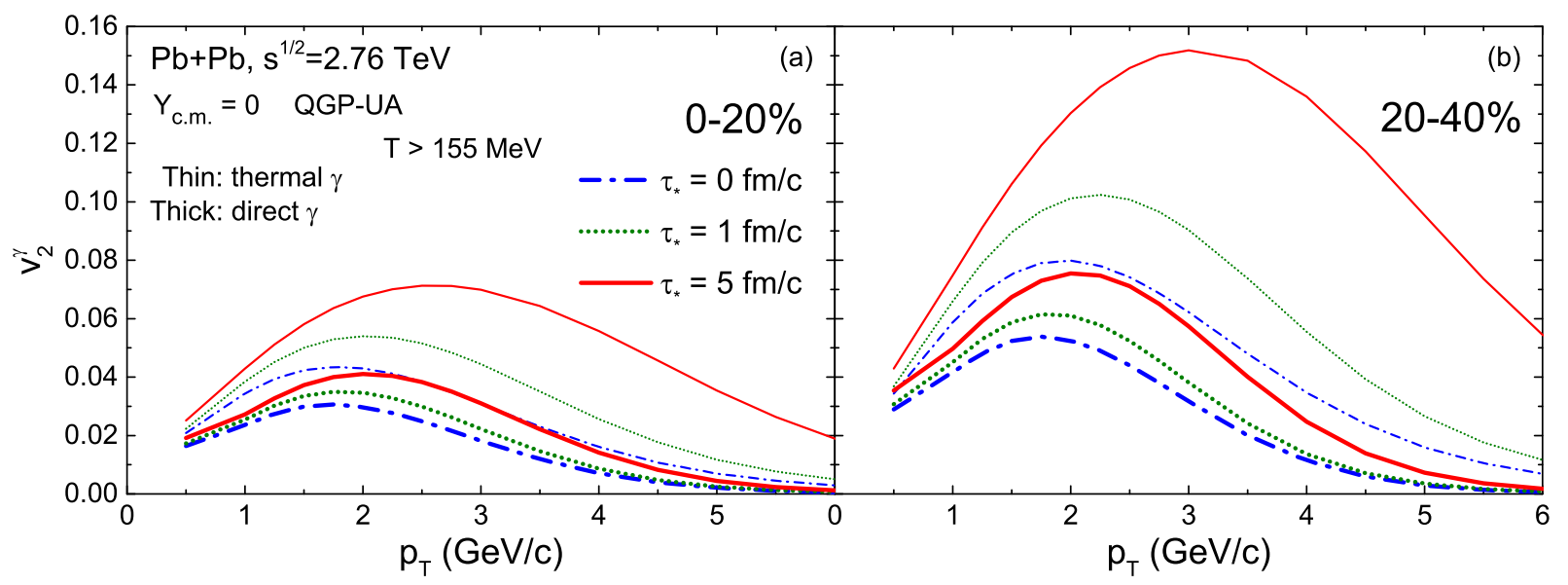

FIG. 10: (Color online) Elliptic flow of the direct (thick lines) and thermal (thin lines) photons for the $0-20 \%$ (a) and $20-40 \%$ (b) central $\mathrm{Pb}+\mathrm{Pb}$ collisions at $\sqrt{s_{\mathrm{NN}}}=2.76 \mathrm{TeV}$.

From the analysis of different cutoff temperatures we conclude that the contribution of a colder 'hadronic' stage increases the photon elliptic flow and leads to a somewhat better agreement with the ALICE data. The latter ones are noticeably underestimated in the chemically equilibrium scenario $\left(\tau_{*}=0\right)$. The physical reason for the $v_{2}^{\gamma}$ increase for lower $T_{f}$ is rather clear. It is explained by the increase of collective flow velocities at later times. Despite the fact that fewer quarks are produced at late stages, their angular anisotropy will be stronger.

To study possible influence of the centrality choice, in Fig. 10 we show the photon elliptic flows for the same reaction, but taking narrower centrality classes, 0-20\% and 20-40\%. One can see that the photon elliptic flow and its sensitivity to chemical nonequilibrium effects becomes stronger for larger impact parameters. This behavior is explained by increased eccentricities of quark fireballs in less central events. 


\section{THERMAL DILEPTON EMISSION}

The rate of thermal dilepton production from the lowest-order quark-antiquark annihilation processes $q \bar{q} \rightarrow e^{+} e^{-}$in the net baryon-free uQGP can be written $\operatorname{as}^{8}$ :

$$
\frac{d N}{d^{4} x d^{4} Q}=C_{q} \lambda^{2} \exp \left(-\frac{Q u}{T}\right)
$$

where $Q=p_{+}+p_{-}$is the dilepton total four-momentum, and $T$ and $u$ are, respectively, the local values of temperature and four-velocity of the medium. The coefficient in front of the rate is $C_{q}=\frac{\alpha^{2}}{4 \pi^{4}} F_{q}$, where $\alpha$ and $F_{q}$ are defined in Eq. (A1). Note that Eq. (16) is obtained in the Boltzmann approximation for the (anti)quark phase-space distributions and neglects the quark and lepton masses. The $\lambda^{2}$ factor in Eq. (16) takes into account the (anti)quark suppression in the chemically nonequilibrated QGP.

Introducing the dilepton invariant mass $M=\sqrt{Q^{2}}$, one has

$$
Q^{\mu}=\left(M_{\perp} \cosh Y, \boldsymbol{Q}_{\perp}, M_{\perp} \sinh Y\right)
$$

where $M_{\perp}=\sqrt{M^{2}+Q_{\perp}^{2}}$ stands for the transverse pair mass, and $Y=\tanh ^{-1}\left(Q_{z} / Q_{0}\right)$ is the longitudinal rapidity of the lepton pair. Using Eq. (3) for the four-velocity of the fluid in the $(2+1)$-dimensional hydrodynamics, one gets the expression for the rest-frame dilepton's total energy

$$
(Q u)=\gamma_{\perp}\left[M_{\perp} \cosh (Y-\eta)-\boldsymbol{Q}_{\perp} \boldsymbol{v}_{\perp}\right]
$$

Let us denote by $\varphi$ and $\varphi_{u}$ the angles of $\boldsymbol{Q}_{\perp}$ and $\boldsymbol{v}_{\perp}$ with respect to the $x$-axis, respectively. Then one can substitute $\boldsymbol{Q}_{\perp} \boldsymbol{v}_{\perp}=Q_{\perp} v_{\perp} \cos \left(\varphi-\varphi_{u}\right)$ in the right-hand side of Eq. (18).

From Eq. (16), using the relations $d^{4} x=\tau d \tau d^{2} x_{\perp} d \eta$ and $d^{4} Q=M d M d Y d^{2} Q_{\perp}$, after integrating over the space-time rapidity $\eta$, we obtain

$$
\frac{d N}{d M^{2} d Y d \varphi}=C_{q} \int d^{2} x_{\perp} \int_{\tau_{0}}^{+\infty} d \tau \tau \lambda^{2}\left(\tau, \boldsymbol{x}_{\perp}\right) J\left(M, \tau, \boldsymbol{x}_{\perp}\right) \theta\left(T-T_{f}\right)
$$

where

$$
J\left(M, \tau, \boldsymbol{x}_{\perp}\right)=\int_{0}^{\infty} d Q_{\perp} Q_{\perp} K_{0}\left(\frac{\gamma_{\perp} M_{\perp}}{T}\right) \exp \left(\frac{\gamma_{\perp} \boldsymbol{Q}_{\perp} \boldsymbol{v}_{\perp}}{T}\right) .
$$

\footnotetext{
8 An analogous expression in the limit of chemically equilibrated plasma $(\lambda=1)$ has been suggested in [29]. First calculations of the dilepton emission in uQGP have been presented in Refs. [17, 28].
} 
Hereinafter we denote by $K_{\nu}(x)$ and $I_{\nu}(x)$ the modified Bessel functions of the order $\nu$. Due to the assumed boost invariance, the dilepton spectrum (19) does not depend on $Y$ within the $(2+1)$-dimensional hydrodynamics. Thus, it should be applied essentially just in the central rapidity region.

Explicit relations for the invariant mass distribution and the elliptic flow of thermal dileptons, obtained from (19) and (20), are given in Eqs. (B1)-(B4) of Appendix B. We would like to emphasize the well known fact that the dilepton mass spectrum does not depend explicitly on the transverse collective velocity $\boldsymbol{v}_{\perp}$ (see (B1) and (B3)). This is to be contrasted with the $p_{T}$-spectra of thermal photons which are given by a superposition of exponents $\exp \left(-p_{T} / T_{\text {eff }}\right)$, where $T_{\text {eff }}$ is the "blue-shifted" effective temperature $T_{\text {eff }}=$ $T \sqrt{\left(1+v_{\perp}\right) /\left(1-v_{\perp}\right)}$.

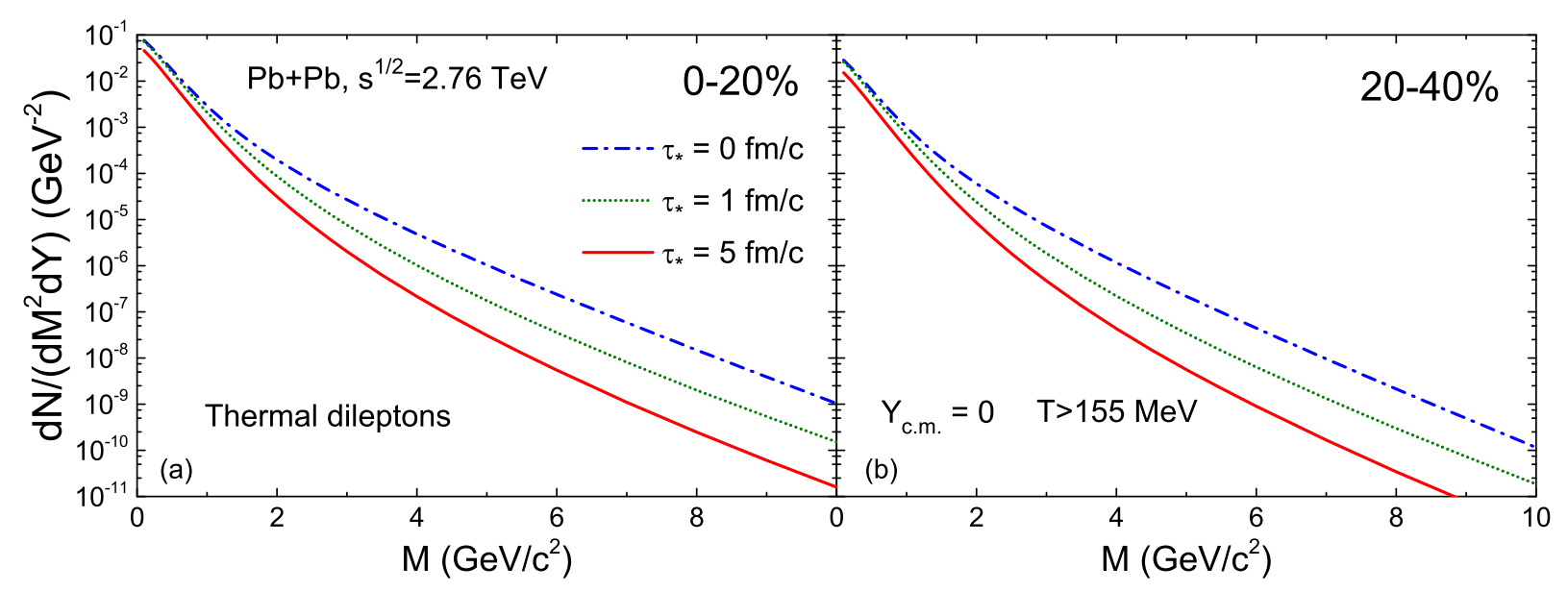

FIG. 11: (Color online) Invariant mass distribution of thermal dileptons in the 0-20\% (a) and $20-40 \%$ (b) central $\mathrm{Pb}+\mathrm{Pb}$ collisions at $\sqrt{s_{N N}}=2.76 \mathrm{TeV}$ calculated for $\tau_{*}=0,1$ and $5 \mathrm{fm} / c$. All results correspond to the cut-off temperature $T_{f}=155 \mathrm{MeV}$.

In the limiting case of the one-dimensional Bjorken-like hydrodynamics one gets for purely central collisions [26]

$$
\frac{d N}{d M^{2} d Y} \simeq 2 \pi^{2} R^{2} C_{q} M \int_{\tau_{0}}^{\tau_{f}} d \tau \tau T(\tau) K_{1}\left[\frac{M}{T(\tau)}\right] \lambda^{2}(\tau) \quad\left(v_{\perp}=0\right)
$$

where $R$ is the geometrical radius of colliding nuclei and $\tau_{f}$ is determined from $T\left(\tau_{f}\right)=T_{f}$.

The results of calculating the dilepton mass spectrum in central $\mathrm{Pb}+\mathrm{Pb}$ collisions at $\sqrt{s_{N N}}=2.76 \mathrm{TeV}$ are shown in Fig. 11 for the cut-off temperature $T_{f}=155 \mathrm{MeV}$. One can 
see that the initial quark suppression leads to a strong reduction of the dilepton yield at $M \gtrsim 2 \mathrm{GeV}$. Note that we do not include contributions of hard (Drell-Yan) dileptons [42] produced in binary collisions of initial nucleons.

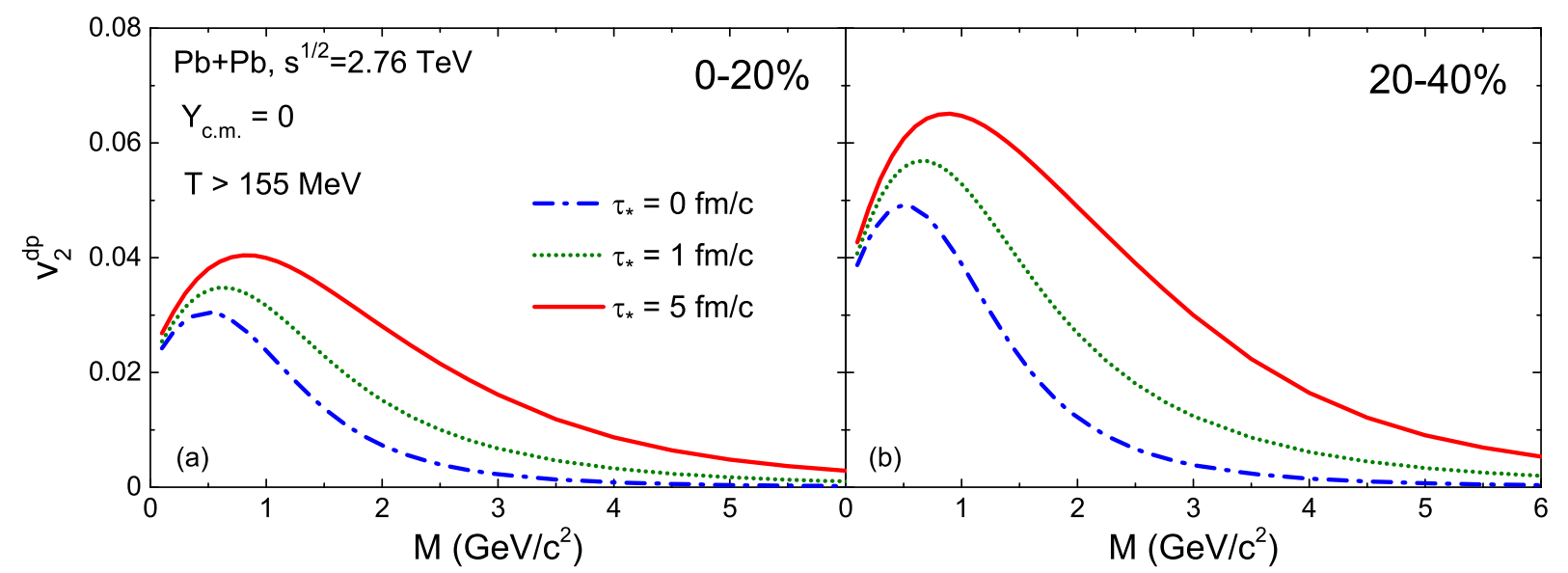

FIG. 12: (Color online) Same as Fig. 11 but for elliptic flow of thermal dileptons $v_{2}^{\mathrm{dp}}$.

As shown in Appendix B, the elliptic flow of thermal dileptons strongly depends both on the direction and magnitude of the transverse collective velocity. Note that the previous analysis of dilepton elliptic flow of Refs. [53, 54] corresponds to the limit of complete chemical equilibrium $(\lambda=1)$.

The elliptic flows of thermal dileptons in the same reaction, calculated in accordance with Eq. (B2), are shown in Fig. 12 for several values of $\tau_{*}$. Similar to direct photons we predict a strong enhancement of the dilepton elliptic flow as compared to the equilibrium scenario $\left(\tau_{*}=0\right)$. Note that $v_{2}^{\mathrm{dp}}$ values are larger for more peripheral events.

\section{CONCLUSIONS}

In this paper we presented calculations of the electromagnetic observables in $\mathrm{Pb}+\mathrm{Pb}$ collisions at LHC energies for different assumptions about the initial state of produced partonic matter. In our calculations we have used a rather advanced hydrodynamic model which was previously used to describe the hadron observables.

In the non-equilibrium scenario, the thermal production of high- $p_{T}$ photons is significantly suppressed compared to the equilibrium case. However, since the high- $p_{T}$ photon production is dominated by the prompt photons from initial parton scatterings, we do not find a strong 
suppression of the total direct photon spectra. Our analysis shows that the $p_{T}$-spectra of such photons calculated for equilibrium and nonequilibrium scenarios differ at most by a factor of 2, and these differences are within the error bars of experimental data. Much stronger effects are found for the thermal dilepton spectra, especially at large invariant masses $M \gtrsim 2 \mathrm{GeV}$, where the deviations between two scenarios can reach one to two orders of magnitude. Unfortunately, the corresponding experimental data are not available yet.

Our hydrodynamic approach also allows us to calculate the elliptic flow parameters $v_{2}^{\gamma, d p}$, which characterize the azimuthal anisotropy of the direct photon and dilepton emission. We find a rather significant enhancement of the elliptic flow for the pure glue initial state for both photons and dileptons. However, the available experimental data for photons are not yet accurate enough to discriminate between the considered scenarios for the initial stage. We are looking forward for the more precise experimental data enabling more definite statements on the evolution of primordial matter in ultrarelativistic heavy-ion collisions.

\section{Acknowledgments}

The authors thank E. L. Bratkovskaya, C. Gale, H. Niemi, L. Pang, and M. Strickland for useful discussions and fruitful comments. This work was partially supported by the Helmholtz International Center for FAIR, Germany, and by the Goal-Oriented Program of the National Academy of Sciences of Ukraine and the European Organization for Nuclear Research (CERN), Grant CO-1-3-2016. L.M.S. and I.N.M. acknowledge a partial support from the grant NSH-932.2014.2 of the Russian Ministry of Education and Science.

\section{Appendix A: Photon Emission}

In this section we consider the PPR in the chemically equilibrated QGP. Processes 1 and 2 (see Sec. [V]) have been analyzed in Ref. [49]. The infrared divergencies of photon production cross sections were regularized by using the hard thermal loop resummation procedure [55]. The following expressions for invariant rates of photon production have been obtained in the lowest order approximation in the strong coupling constant $\alpha_{s}$ :

$$
\Gamma_{i}(\widetilde{E}, T) \equiv E \frac{d N_{i}}{d^{3} p d^{4} x}=A_{i} F_{q} \alpha \alpha_{s} T^{2} e^{-x} \ln \frac{B_{i} x}{\alpha_{s}} \quad(i=1,2) .
$$


Here $p^{\mu}=(E, \boldsymbol{p})^{\mu}$ is the photon four-momentum, $\widetilde{E}=p_{\mu} u^{\mu}$ is the rest-frame photon energy, $\alpha=e^{2} \simeq 1 / 137$ is the electromagnetic coupling constant, $F_{q}=\sum_{f}\left(\frac{e_{f}}{e}\right)^{2}\left(e_{f}\right.$ is the charge of quarks with flavor $f$ ), and $x=\widetilde{E} / T$. Numerical values of constants $A_{i}, B_{i}$ are given by the relations

$$
A_{2}=2 A_{1}=\frac{1}{3 \pi^{2}}, \quad B_{1} \simeq 1.00, \quad B_{2} \simeq 0.112 .
$$

In the following we assume the number of quark flavours $N_{f}=3$ and take into account the temperature dependence of $\alpha_{\mathcal{S}}$ by using the parametrization [56]

$$
\alpha_{s}=\frac{6 \pi}{\left(33-2 N_{f}\right) \ln \left(8 T / T_{*}\right)},
$$

where $T_{*}=170 \mathrm{MeV}$.

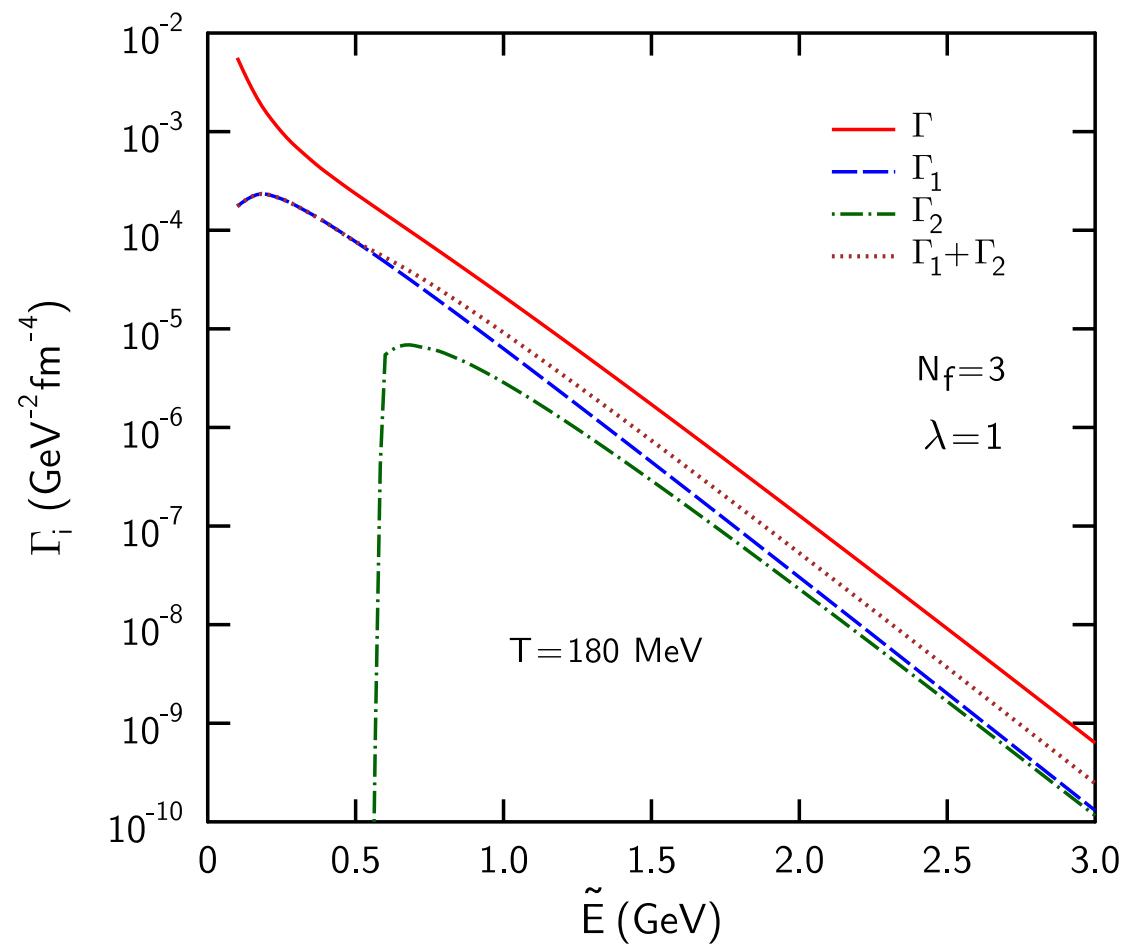

FIG. 13: (Color online) Thermal photon production rates in equilibrium QGP as functions of the rest-frame photon energy at temperature $T=180 \mathrm{MeV}$.

Processes 3 and 4 correspond to higher orders in $\alpha_{s}$. The detailed calculations in [44] give the following result for the total PPR:

$$
\begin{aligned}
& \Gamma(\widetilde{E}, T)=\sum_{i=1}^{4} \Gamma_{i}(\widetilde{E}, T)=\frac{1}{\pi^{2}} F_{q} \alpha \alpha_{s} T^{2} \Phi(x), \\
& \Phi(x)=\left(e^{x}+1\right)^{-1}\left[\frac{1}{2} \ln \frac{3 x}{2 \pi \alpha_{s}}+C_{12}(x)+C_{34}(x)\right],
\end{aligned}
$$


where

$$
C_{12}(x)=0.041 x^{-1}-0.3615+1.01 e^{-1.35 x},
$$

and

$$
C_{34}(x)=\sqrt{1+\frac{N_{f}}{6}}\left[\frac{0.548}{x^{3 / 2}} \ln \left(12.28+x^{-1}\right)+\frac{0.133 x}{\sqrt{1+x / 16.27}}\right] .
$$

These formulas become not accurate outside the domain $0.2 \lesssim x \lesssim 50$. Figure 13 shows

numerical values of $\Gamma_{1}, \Gamma_{2}$, and $\Gamma$ for $T=180 \mathrm{MeV}$. One can see that contributions of processes 3 and 4 are rather significant at all considered photon energies.

\section{Appendix B: Dilepton Emission}

The invariant mass spectrum $d N / d M^{2} d Y$ and the elliptic flow $v_{2}=v_{2}(M)$ of thermal dileptons are determined by integrating Eq. (19) over $\varphi$ with the weights 1 and $\cos (2 \varphi)$, respectively. We get the relations

$$
\begin{gathered}
\frac{d N}{d M^{2} d Y}=C_{q} \int d^{2} x_{\perp} \int_{\tau_{0}}^{+\infty} d \tau \tau \lambda^{2}\left(\tau, \boldsymbol{x}_{\perp}\right) J_{1}\left(M, \tau, \boldsymbol{x}_{\perp}\right) \theta\left(T-T_{f}\right), \\
v_{2} \frac{d N}{d M^{2} d Y}=C_{q} \int d^{2} x_{\perp} \int_{\tau_{0}}^{+\infty} d \tau \tau \lambda^{2}\left(\tau, \boldsymbol{x}_{\perp}\right) J_{2}\left(M, \tau, \boldsymbol{x}_{\perp}\right) \theta\left(T-T_{f}\right),
\end{gathered}
$$

where

$$
J_{1}=\int_{0}^{2 \pi} d \varphi J=2 \pi \int_{0}^{\infty} d Q_{\perp} Q_{\perp} K_{0}\left(\frac{\gamma_{\perp} M_{\perp}}{T}\right) I_{0}\left(\frac{\gamma_{\perp} v_{\perp} Q_{\perp}}{T}\right)=2 \pi M T K_{1}\left(\frac{M}{T}\right)
$$

and

$$
\begin{aligned}
J_{2}=\int_{0}^{2 \pi} d \varphi J \cos (2 \varphi) & =2 \pi \cos \left(2 \varphi_{u}\right) \int_{0}^{\infty} d Q_{\perp} Q_{\perp} K_{0}\left(\frac{\gamma_{\perp} M_{\perp}}{T}\right) I_{2}\left(\frac{\gamma_{\perp} v_{\perp} Q_{\perp}}{T}\right) \\
& =\cos \left(2 \varphi_{u}\right)\left\{J_{1}-\frac{4 \pi T^{2}}{\gamma_{\perp}^{2}-1}\left[K_{0}\left(\frac{M}{T}\right)-K_{0}\left(\frac{\gamma_{\perp} M}{T}\right)\right]\right\} .
\end{aligned}
$$

To calculate integrals over $Q_{\perp}$ in Eqs. (B3) and (B4), we have applied a procedure suggested in [57]. We start from the integral representation

$$
K_{\nu}(x)=x^{\nu} \int_{0}^{\infty} \frac{d t}{t^{\nu+1}} \exp \left[-\frac{1}{2}\left(t+\frac{x^{2}}{t}\right)\right]
$$


and then use the formulas

$$
\begin{aligned}
& \int_{0}^{\infty} d Q_{\perp} Q_{\perp} e^{-A Q_{\perp}^{2}} I_{0}\left(B Q_{\perp}\right)=\frac{1}{2 A} \exp \left(\frac{B^{2}}{4 A}\right), \\
& \int_{0}^{\infty} d Q_{\perp} Q_{\perp} e^{-A Q_{\perp}^{2}} I_{2}\left(B Q_{\perp}\right)=\left(\frac{1}{2 A}-\frac{2}{B^{2}}\right) \exp \left(\frac{B^{2}}{4 A}\right)+\frac{2}{B^{2}} .
\end{aligned}
$$

The second equation is obtained by using the relation $I_{2}(x)=I_{0}(x)-2 I_{0}^{\prime}(x) / x$.

Note that $\cos \left(2 \varphi_{u}\right)=\left(v_{x}^{2}-v_{y}^{2}\right) / v_{\perp}^{2}$ in Eq. (B) . It is easy to show that at small $v_{\perp}$ one gets the approximate relation

$$
J_{2} \simeq \frac{\pi}{2}\left(v_{x}^{2}-v_{y}^{2}\right) M^{2} K_{2}\left(\frac{M}{T}\right) \quad\left(v_{\perp} \ll 1\right) .
$$

Presumably, this limiting case corresponds to early stages of the QGP evolution.

[1] X.-N. Wang, M. Gyulassy, Phys. Rev. D 44, 3501 (1991).

[2] Z. Xu and C. Greiner, Phys. Rev. C 71, 064901 (2005).

[3] S. A. Bass et al., Prog. Part. Nucl. Phys. 41, 225 (1998).

[4] M. Bleicher et al., J. Phys. G25, 1859 (1999).

[5] W. Cassing and E. L. Bratkovskaya, Phys. Rev. C 78, 034919 (2008);

Nucl. Phys. A 831, 215 (2009).

[6] L. D. McLerran, R. Venugopalan, Phys. Rev. D 49, 2233 (1994).

[7] V. K. Magas, L. P. Csernai, and D. D. Strottman, Phys. Rev. C 64, 014901 (2001).

[8] I. N. Mishustin, J. I. Kapusta, Phys. Rev. Lett. 88, 112501 (2002).

[9] B. Schenke, P. Tribedy, and R. Venugopalan, Phys. Rev. Lett. 108, 252301 (2012).

[10] L. V. Gribov, E. M. Levin, M. G. Ryskin, Phys. Rept. 100, 1 (1983).

[11] L. van Hove and S. Pokorski, Nucl. Phys. B 86, 243 (1975).

[12] S. Raha, Physica Scripta T32, 180 (1990).

[13] E. Shuryak, Phys. Rev. Lett. 68, 3270 (1992).

[14] J. Alam, S. Raha, and B. Sinha, Phys. Rev. Lett. 73, 1895 (1994).

[15] T. S. Biró, E. van Doorn, B. Müller, M. H. Thoma, and X.-N. Wang, Phys. Rev. C 48, 1275 (1993).

[16] B. Kämpfer and O. P. Pavlenko, Z. Phys. C 62, 491 (1994). 
[17] B. Kämpfer, O. P. Pavlenko, A. Peshier, and G. Soff, Phys. Rev. C 52, 2704 (1995).

[18] C. T. Traxler, M. H. Thoma, Phys. Rev. C 53, 1348 (1996).

[19] D. M. Elliott, D. H. Rischke, Nucl. Phys. A 671, 583 (2000).

[20] D. Dutta, S.V.S. Sastry, A. K. Mohanty, K. Kumar, and R. K. Choudhury, Nucl. Phys. A 710, 415 (2002).

[21] F. Gelis, H. Niemi, R. V. Ruuskanen, S. S. Räsänen, J. Phys. G: Nucl. Part. Phys. 30, S1031 (2004).

[22] F.-M. Liu and S.-X. Liu, Phys. Rev. C 89, 034906 (2014).

[23] A. Monnai, Phys. Rev. C 90, 021901 (2014).

[24] P. Moreau, O. Linnyk, W. Cassing, and E.L. Bratkovskaya, Phys. Rev. C 93, 044916 (2016).

[25] H. Stoecker et al., J. Phys. G43, 015105 (2016).

[26] H. Stöcker et al., Astron. Nachr. 336, 744 (2015).

[27] V. Vovchenko, M. I. Gorenstein, L. M. Satarov, I. N. Mishustin, L. P. Csernai, I. Kisel, and H. Stöcker, Phys. Rev. C 93, 014906 (2016).

[28] M. Strickland, Phys. Lett. B 331, 245 (1994).

[29] K. Kajantie, M. Kataja, L. M. McLerran, and P. V. Ruuskanen, Phys. Rev. D 34, 811 (1986).

[30] J. D. Bjorken, Phys. Rev. D 27, 140 (1983).

[31] M. Ruggieri, S. Plumari, F. Scardina, and V. Greco, Nucl. Phys. A 941, 201 (2015).

[32] S. Borsanyi, Z. Fodor, C. Hoelbling, S. D. Katz, S. Krieg, and K. K. Szabo, Phys. Lett. B 730, 99 (2014).

[33] A. Bazavov (HotQCD Collaboration), Nucl. Phys. A 931, 867 (2014).

[34] G. Boyd, J. Engels, F. Karsch, E. Laermann, C. Legeland, M. Lutgemeier, and B. Petersson, Phys. Rev. Lett. 75, 4169 (1995).

[35] S. Borsanyi, G. Endrodi, Z. Fodor, S. D. Katz and K. K. Szabo, JHEP 1207, 056 (2012).

[36] W. Broniowski, M. Rybczynski, and P. Bozek, Comput. Phys. Commun. 180, 69 (2009).

[37] I. A. Karpenko, Y. M. Sinyukov, and K. Werner, Phys. Rev. C 87, 024914 (2013).

[38] I. Karpenko, P. Huovinen, and M. Bleicher, Comput. Phys. Commun. 185, 3016 (2014).

[39] B. Abelev et al. (ALICE Collaboration), Phys. Rev. C 88, 044910 (2013).

[40] L. M. Satarov, I. N. Mishustin, A. V. Merdeev, and H. Stöcker, Phys. Atom. Nucl. 70, 1773 (2007); Phys. Rev. C 75, 024903 (2007).

[41] J. F. Paquet, C. Shen, G. S. Denicol, M. Luzum, B. Schenke, S. Jeon, and C. Gale, 
Phys. Rev. C 93, 044906 (2016).

[42] O. Linnyk, E. L. Bratkovskaya, W. Cassing, Prog. Part. Nucl. Phys. 87, 50 (2016).

[43] D. Lohner [ALICE Collaboration], J. Phys. Conf. Ser. 446, 012028 (2013)

[44] P. Arnold, G. D. Moore, and L. G. Yaffe, JHEP 12, 009 (2001).

[45] J. Ghiglieri, J. Hong, A. Kurkela, E. Lu, G. D. Moore, and D. Teaney, JHEP 05, 010 (2013).

[46] J. Adam et al. [ALICE Collaboration], Phys. Lett. B 754, 235 (2016).

[47] M. Heffernan, P. Hohler, and R. Rapp, Phys. Rev. C 91, 027902 (2015).

[48] S. Turbide, R. Rapp, and C. Gale, Phys. Rev. C 69, 014903 (2004).

[49] J. Kapusta, P. Lichard, D. Seibert, Phys. Rev. D 44, 2774 (1991).

[50] R. Chatterjee, H. Holopainen, T. Renk, and K. J. Eskola, Phys. Rev. C 85, 064910 (2012).

[51] H. van Hees, M. He, and R. Rapp, Nucl. Phys. A 933, 256 (2015).

[52] O. Linnyk, V. Konchakovski, T. Steinert, W. Cassing, and E. L. Bratkovskaya, Phys. Rev. C 92, 054914 (2015).

[53] R. Chatterjee, D. K. Srivastava, U. Heinz, and C. Gale, Phys. Rev. C 75, 054909 (2007).

[54] G. Vujanovic et al., arXiv: 1602.01455.

[55] R. D. Pisarski, Nucl. Phys. B 309, 476 (1988); Phys. Rev. Lett. 63, 1129 (1989).

[56] F. D. Steffen and M. H. Thoma, Phys. Lett. B 510, 98 (2001).

[57] G. N. Watson, A Treatese on the Theory of Bessel Functions, (Cambridge University Press, Cambridge, 1995), p. 415. 